\title{
Communication
}

\section{Free Radical-Mediated Damage of Blood and Its Inhibition by Antioxidants}

\author{
Etsuo NiKI, Yorihiro Yamamoto, Mareyuki TAKAHASHI, \\ Keiji YAMAMOTO, Yuichi YAMAMOTO, Erika KomURo, ${ }^{1}$ \\ Masayuki MIKI, Hiroshi YASUdA, and Makoto MINO ${ }^{2}$ \\ ${ }^{1}$ Department of Reaction Chemistry, Faculty of Engineering, \\ University of Tokyo, Bunkyo-ku, Tokyo 113, Japan \\ ${ }^{2}$ Department of Pediatrics, Osaka Medical College, \\ Takatsuki, Osaka 569, Japan
}

(Received July 27, 1988)

Key Words free radical, blood, oxidation, antioxidants, hemolysis

The formation, behavior, and scavenging of free radicals in biological systems have received much attention with increasing evidence that they are closely connected with a variety of pathological events, cancer, and aging $(1)$. For the quantitative study on oxidative damage of biological systems, it is essential to generate a definite free radical in a known place and at a constant rate. A watersoluble radical initiator, 2,2'-azobis(2-amidinopropane) dihydrochloride (AAPH), is suitable for this purpose and has been used recently in in vitro (2-9) and even in vivo study (10). We have shown that peroxy radicals derived from AAPH attack erythrocytes, induce the chain oxidations of membrane lipids and proteins, and eventually cause hemolysis $(5,6)$. These damages of erythrocytes were inhibited by exogenous antioxidants such as ascorbic acid, uric acid, cysteine, and glutathione(7). Ingold and his colleagues (8) measured the total radical-trapping capacity of antioxidants in human plasma and found that their contributions were $35-65 \%$ from urate, $0-24 \%$ from ascorbate, $5-10 \%$ from vitamin $E$, and $10-50 \%$ from plasma proteins. They also observed that sulfhydryl groups of proteins decreased firstly, even urate and vitamin $\mathrm{E}$ being spared at the initial stages. Stocker et al. (9) observed that the antioxidants reacted with peroxy radicals derived from AAPH in the order of ascorbate $>$ albumin-bound bilirubin $>$ urate. It is also known that vitamin $\mathrm{E}$ functions as an antioxidant in the erythrocyte membranes (4-6). However, it has not been known how these antioxidants behave against free radicals in the whole blood. In the present study, the fate and consequence of the

\footnotetext{
${ }^{1}$ 二木鋭雄，山本順寛，高橋希之，山本敬司，山本裕一，小室絵里佳， ${ }^{2}$ 三木正之,

2 保田 浩, 美濃 真
} 
free radicals and also the behavior of various antioxidants have been studied for the first time in the AAPH-initiated oxidation of the whole blood. We believe that this system serves as a model for the oxidative damage that will take place when free radicals are generated in the blood and gives us information how the various endogenous antioxidants in the blood function against the free radicals.

The heparinized blood obtained from healthy donors or its aqueous dilution was incubated aerobically in the presence and absence of AAPH (Wako Pure Chemical, Osaka) under constant shaking in a water bath maintained at $37^{\circ} \mathrm{C}$. The extent of hemolysis was measured spectrophotometrically as reported previously (5). An aliquot was taken out periodically, centrifuged at $10,000 \times g$ for 5 min to separate plasma and erythrocytes. Ascorbic and uric acids were measured by HPLC equipped with an electrochemical detector (Model ECP-1, Kotaki, Chiba) using ODS column and $0.2 \mathrm{M}$ phosphate buffer ( $\mathrm{pH} 2.0$ ) containing $1 \mathrm{mM}$ EDTA as eluent (11). $\alpha$-Tocopherol in both plasma and erythrocytes was also measured by HPLC equipped with an electrochemical detector(12) after the extraction with hexane. Sulfhydryl groups in the erythrocyte ghost membranes were measured by a reported method(13). Neutral lipid hydroperoxides which can be extracted into hexane and plasma levels of ascorbic acid, uric acid, and bilirubin were measured by a reported method(14). Plasma levels of lactate dehydrogenase (LDH), aspartate transaminase (GOT), potassium ion, and calcium ion were assayed at Teijin BioScience Laboratories (Tokyo).

As shown in Fig. 1, AAPH induced the hemolysis of erythrocytes dosedependently. In the absence of AAPH, little hemolysis was observed in $400 \mathrm{~min}$ under the present condition (Runs 1 and 4). Furthermore, the leakage of hemoglobin was not observed when $50 \mathrm{~mm}$ of AAPH was added to the whole blood (Run 2) nor when whole blood was diluted with three volumes of physiological saline and oxidized with $30 \mathrm{~mm}$ of AAPH (Run 3). On the other hand, when $50 \mathrm{~mm}$ of AAPH was added to whole blood diluted with physiological saline by a factor of 10 (Run 5), the hemolysis took place in about $250 \mathrm{~min}$. The hemolysis took place in about $100 \mathrm{~min}$ when whole blood was put into three volumes of AAPH aqueous solution; final concentration of AAPH was $200 \mathrm{~mm}$ (Run 6). Along with the hemolysis the leakages of GOT, LDH, and calcium ion were observed (data not shown). The erythrocyte membranes must be attacked by peroxy radicals and suffer more damage with increasing number of attacking peroxy radicals as observed in the AAPH-initiated oxidations of the erythrocyte membranes $(5,6)$ and ghost membranes (4) in the absence of plasma.

Figure 2 shows the results obtained when whole blood was diluted with AAPH solution by a factor of 4 and oxidized at $37^{\circ} \mathrm{C}$ under air; final concentration of AAPH was $200 \mathrm{~mm}$ (Run 6). Ascorbic acid and bilirubin disappeared within $10 \mathrm{~min}$ (data not shown). Uric acid and $\alpha$-tocopherol also decreased rapidly and disappeared. Neutral lipid hydroperoxides in the plasma increased soon after the start of incubation. $\alpha$-Tocopherol and sulfhydryl groups in the erythrocyte membrane remained unchanged at the initial stage, but they began to decrease after about 80 - 


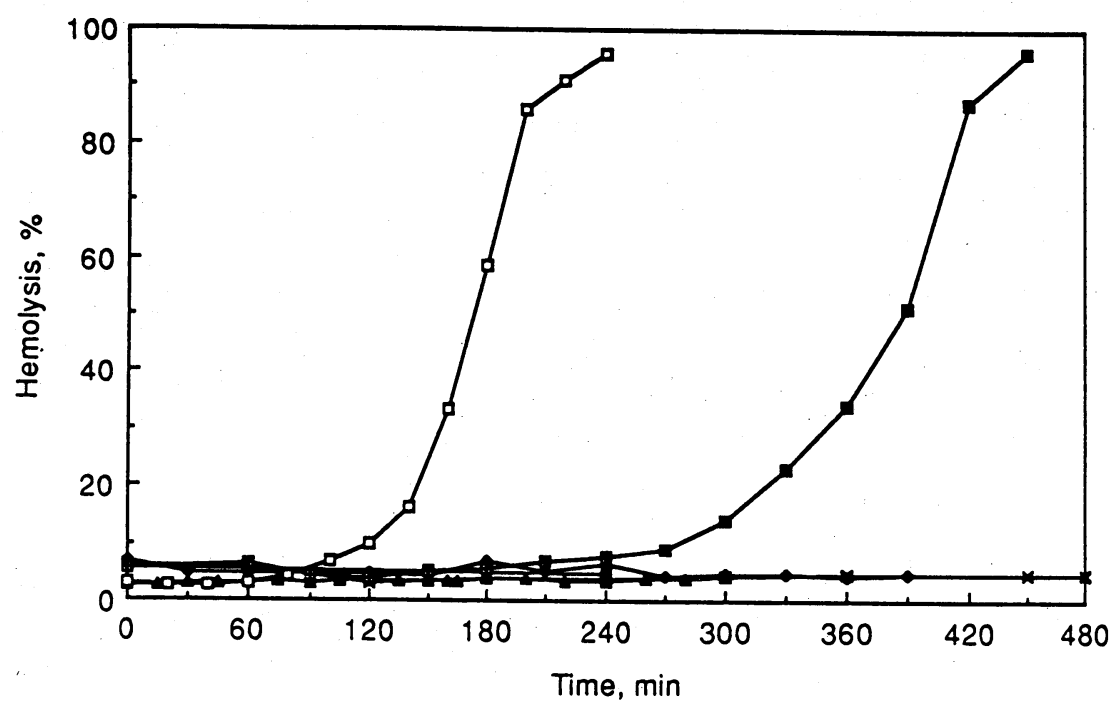

Fig. 1. Hemolysis in the oxidation of the whole human blood initiated with AAPH at $37^{\circ} \mathrm{C}$ under air.

\begin{tabular}{cccc}
\hline Run & Symbol & Factor of dilution & AAPH, mM \\
\hline 1 & & 1 (whole blood) & 0 \\
2 & - & 1 (whole blood) & 50 \\
3 & $\mathbf{4}$ & 4 with physiological saline & 30 \\
4 & $\times$ & 10 with physiological saline & 0 \\
5 & 10 with physiological saline & 50 \\
6 & $\square$ & 4 with AAPH solution & 200 \\
\hline
\end{tabular}

min incubation. The leakages of hemoglobin, LDH, GOT, and calcium ion took place in about $100 \mathrm{~min}$ after the considerable amounts of $\alpha$-tocopherol and sulfhydryl groups in the erythrocyte membrane were consumed. On the other hand, potassium ion leaked out from a very early stage.

Figure 3 shows the results of oxidation of blood under milder conditions; that is, whole blood diluted four times with physiological saline was oxidized with $30 \mathrm{mM}$ AAPH (Run 3). AAPH was added $60 \mathrm{~min}$ after the start of the incubation at $37^{\circ} \mathrm{C}$ under air. Ascorbic acid disappeared soon after the addition of AAPH, and bilirubin, $\alpha$-tocopherol, and uric acid in the plasma were also consumed rapidly in this order. On the other hand, erythrocyte membrane $\alpha$-tocopherol was not consumed appreciably and level of membrane sulfhydryl groups did not change either. The leakages of hemoglobin, GOT, LDH, and calcium ion were not observed, but potassium ion gradually leaked out after about 220 -min incubation. 


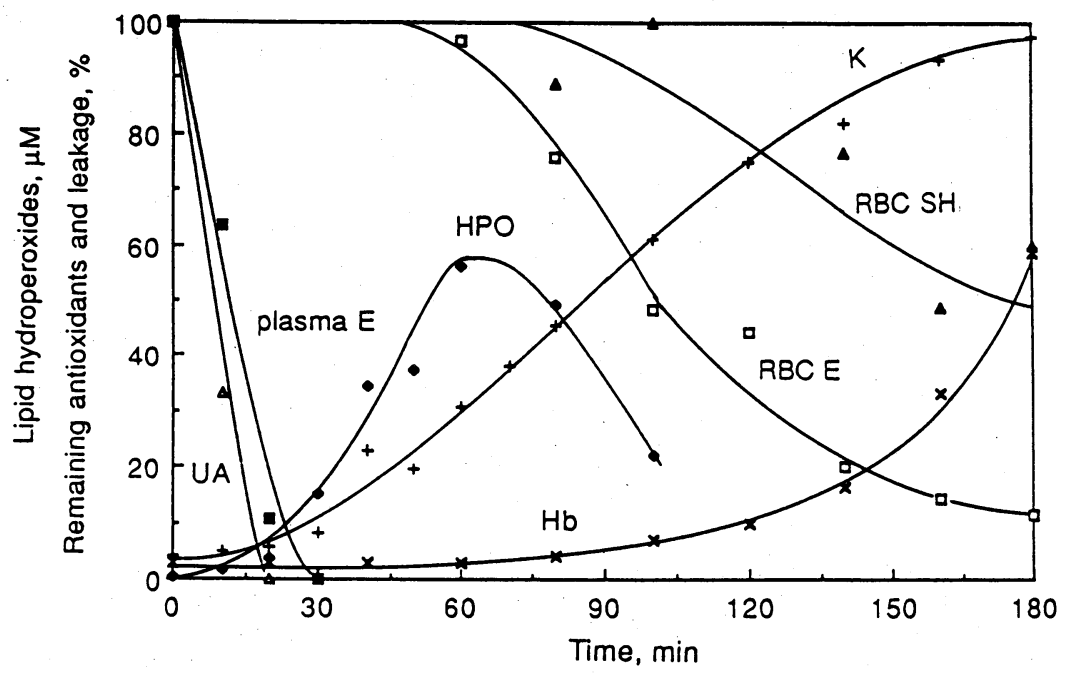

Fig. 2. Decrease of uric acid (UA, $\triangle$ ), plasma $\alpha$-tocopherol (plasma E, $\boldsymbol{\square}$ ), membrane $\alpha$-tocopherol (RBC E, $\square$ ), and membrane sulfhydryl groups (RBC SH, $\mathbf{\Delta}$ ), formation of neutral lipid hydroperoxides (HPO, $\bullet$ ), and leakages of hemoglobin $(\mathrm{Hb}, \times)$ and potassium ion $(\mathrm{K},+)$ in the AAPH-initiated oxidation of 4-times diluted whole blood with $200 \mathrm{~mm}$ AAPH at $37^{\circ} \mathrm{C}$ under air.

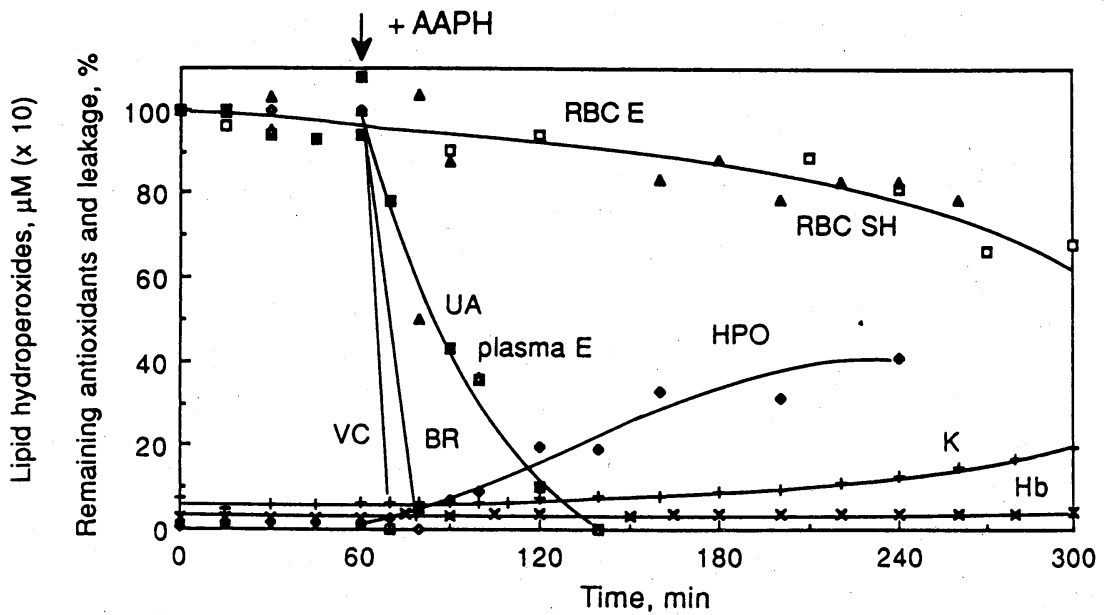

Fig. 3. Decrease of ascorbic acid (VC, $⿴ 囗 十)$, bilirubin (BR, $\diamond)$, uric acid (UA, $\triangle$ ), plasma $\alpha$-tocopherol (plasma E, $\boldsymbol{0}$ ), membrane $\alpha$-tocopherol (RBC E, $\square$ ), and membrane sulfhydryl groups (RBC SH, $\Delta$ ), formation of neutral lipid hydroperoxides (HPO, $\diamond)$, and leakages of hemoglobin $(\mathrm{Hb}, \times)$ and potassium ion $(\mathrm{K},+)$ in the oxidation of 4-times diluted human whole blood with physiological saline initiated with $30 \mathrm{~mm} \mathrm{AAPH}$ at $37^{\circ} \mathrm{C}$ under air. 
On the other hand, the increase of neutral lipid hydroperoxides had been observed since 80-min incubation.

Figures 2 and 3 show that antioxidants decreased in the order of ascorbic acid, bilirubin, uric acid, plasma $\alpha$-tocopherol, membrane $\alpha$-tocopherol, and membrane sulfhydryl groups. This is consistent with the earlier findings by Stocker et al. (9) and Wayner et al. (8); that is, albumin-bound bilirubin reacts with peroxy radicals from AAPH slower than ascorbic acid but faster than uric acid, and uric acid decreases faster than plasma $\alpha$-tocopherol. However, the order of reactivity of plasma and membrane antioxidants has not been measured before. Our results clearly show that plasma antioxidants especially ascorbic acid are the first defense lines against the peroxy radicals formed in the plasma. When ascorbic acid was depleted and a considerable amount of plasma $\alpha$-tocopherol was consumed, the neutral lipid hydroperoxides in the plasma began to increase markedly.

As shown in Fig. 2, potassium ion leaked out much earlier than the release of hemoglobin and the consumption of membrane $\alpha$-tocopherol and sulfhydryl groups, indicating that the damage of erythrocyte membrane took place at the early stage of oxidation. Chiu et al.(15) and Tanaka et al.(16) also observed the same order of leakages in the hydrogen peroxide-initiated oxidation of vitamin Edeficient erythrocytes from patients with sickle cell anemia and in the interaction between short-chain phosphatidylcholine and erythrocytes, respectively. On the other hand, Deuticke et al. (17) reported that the leak of ions was induced by the crosslinking of spectrin via disulfide bridges formed in the presence of diamide. Crosslinking of proteins may be critical for the leakage of potassium ion, since oxidative crosslinking of proteins was observed in the oxidation of ghosts with AAPH $(4)$ or organic hydroperoxides $(18,19)$ and in the oxidation of erythrocytes with AAPH (6). At any event, the leak of potassium ion can be a good indicator of oxidative damage of erythrocyte membrane at the early stage, although the precise mechanism is not known.

Finally, it may be noteworthy that this study does not necessarily deny the vital role of vitamin E in vivo. In the present study, the radicals were generated initially in the plasma or in the aqueous region, and hydrophilic antioxidants functioned efficiently. However, these antioxidants are not capable of scavenging the radicals within the membrane and only vitamin $\mathrm{E}$ can scavenge them and must function as a last defense. Furthermore, if the radicals are generated initially within the membrane, the vitamin $\mathrm{E}$ must be the primary and only defense.

In conclusion, this study shows that vitamin $\mathrm{C}$ and vitamin $\mathrm{E}$ function as primary hydrophlic and lipophilic chain-breaking antioxidants, respectively, in the blood.

\section{REFERENCES}

1) For example, Pryor, W. A., ed. (1976-1984): Free Radicals in Biology, Vol. I-VI, Academic Press, New York.

2) Yamamoto, Y., Haga, S., Niki, E., and Kamiya, Y. (1984): Oxidation of methyl

Vol. 34 , No. 5, 1988 
linoleate in aqueous dispersion. Bull. Chem. Soc. Jpn., 57, 1260-1264.

3) Barclay, L. R. C., Locke, S. J. MacNeil, J. M., Burton, G. W., and Ingold, K. U. (1984): Autoxidation of micelles and model membranes. Quantitative kinetic measurements can be made by using either water-soluble or lipid-soluble initiators with water-soluble or lipid-soluble chain-breaking antioxidants. J. Am. Chem. Soc., 106, 2479-2481.

4) Yamamoto, Y., Niki, E., Eguchi, J., Kamiya, Y., and Shimasaki, H. (1985): Free radical chain oxidation of erythrocyte ghost membranes and its inhibition. Biochim. Biophys. Acta, 819, 29-36.

5) Yamamoto, Y., Niki, E., Kamiya, Y., Miki, M., Tamai, H., and Mino, M. (1986): Free radical chain oxidation and hemolysis of erythrocytes by molecular oxygen and their inhibition by vitamin E. J. Nutr. Sci. Vitaminol., 32, 475-479.

6) Miki, M., Tamai, H., Mino, M., Yamamoto, Y., and Niki, E. (1987): Free-radical chain oxidation of rat red blood cells by molecular oxygen and its inhibition by $\alpha$-tocopherol. Arch. Biochem. Biophys., 258, 373-380.

7) Niki, E. (1987): Antioxidants in relation to lipid peroxidation. Chem. Phys. Lipids, 44, 227-253.

8) Wayner, D. D. M., Burton, G. W., Ingold, K. U., Barclay, L. R. C., and Locke, S. (1987): The relative contributions of vitamin E, urate, ascorbate and proteins to the total peroxyl radical-trapping antioxidant activity of human blood plasma. Biochim. Biophys. Acta, 924, 408-419.

9) Stocker, R., Glazer, A. N., and Ames, B. N. (1987): Antioxidant activity of albuminbound bilirubin. Proc. Natl. Acad. Sci. U.S.A., 84, 5918-5922.

10) Terao, K., and Niki, E. (1986): Damage to biological tissues induced by radical initiator 2,2'-azobis(2-amidinopropane) dihydrochloride and its inhibition by chain-breaking antioxidants. J. Free Radical Biol. Med., 2, 193-201.

11) Yoshiura, M., Iwamoto, T., and Iriyama, K. (1984): Attempt at the determination of ascorbic acid by reversed-phase high-performance liquid chromatography with electrochemical detection. Jikeikai Med. J., 31, 85-91.

12) Yamamoto, K., and Niki, E. (1988): Interaction of $\alpha$-tocopherol with iron: antioxidant and prooxidant effects of $\alpha$-tocopherol in the oxidation of lipids in aqueous dispersions in the presence of iron. Biochim. Biophys. Acta, 958, 19-23.

13) Fernandes Diez, M. J., Osuga, D. T., and Feexey, R. E. (1964): The sulfhydryls of avian ovalbumin, bovine $\beta$-lactoglobulin, and bovine serum albumin. Arch. Biochem. Biophys., 107, 449-458.

14) Yamamoto, Y., and Ames, B. N. (1987): Detection of lipid hydroperoxides and hydrogen peroxide at picomole levels by an HPLC and isoluminol chemiluminescence assay. Free Radical Biol. Med., 3, 359-361.

15) Chiu, D., Vichinsky, E., Yee, M., Kleman, K., and Lubin, B. (1982): Peroxidation, vitamin E, and sickle-cell anemia. Ann. N. Y. Acad. Sci., 393, 323-335.

16) Tanaka, Y., Inoue, K., and Nojima, S. (1980): Interaction of dilauroylglycerophosphocholine with erythrocytes. Biochim. Biophys. Acta, 600, 126-139.

17) Deuticke, B., Lutkemeier, P., and Sistemich, M. (1984): Ion selectivity of aqueous leaks induced in the erythrocyte membrane by crosslinking of membrane proteins. Biochim. Biophys. Acta, 775, 150-160.

18) Koster, J. F., and Slee, R. G. (1983): Lipid peroxidation of human erythrocyte ghosts induced by organic hydroperoxides. Biochim. Biophys. Acta, 752, 233-239.

19) Beppu, M., Murakami, K., and Kikugawa, K. (1987): Detection of oxidized lipidmodified erythrocyte membrane proteins by radiolabeling with tritiated borohydride. Biochim. Biophys. Acta, 897, 169-179. 